\title{
THE CESÀRO AND RELATED OPERATORS, A SURVEY
}

\author{
V. G. MILLER \\ Department of Mathematics and Statistics, Mississippi State University \\ Drawer MA, Mississippi State, MS 39762, U.S.A. \\ E-mail: vivien@math.msstate.edu
}

\begin{abstract}
We provide a survey of properties of the Cesàro operator on Hardy and weighted Bergman spaces, along with its connections to semigroups of weighted composition operators. We also describe recent developments regarding Cesàro-like operators and indicate some open questions and directions of future research.
\end{abstract}

1. Introduction and history. If $U$ is an open subset of the complex plane $\mathbb{C}$, let $\mathcal{H}(U)$ denote the space of complex-valued analytic functions on $U$, a Fréchet algebra when endowed with the topology of uniform convergence on compact subsets of $U$. Let $\mathbb{D}$ denote the open unit disk in $\mathbb{C}$ and, for $f \in \mathcal{H}(\mathbb{D})$ with power series expansion $f(z)=\sum_{n=0}^{\infty} a_{n} z^{n}$, define

$$
C f(z)=\sum_{n=0}^{\infty} \frac{1}{n+1}\left(\sum_{k=0}^{n} a_{k}\right) z^{n} .
$$

The operator $C$ may also be represented as an integral operator:

$$
C f(z)=\frac{1}{z} \int_{0}^{z} \frac{f(\omega)}{1-\omega} d \omega
$$

and in this form, it is easy to see that $C: \mathcal{H}(\mathbb{D}) \rightarrow \mathcal{H}(\mathbb{D})$ is continuous. The classical Hardy space $H^{2}(\mathbb{D})$ consists of those function in $\mathcal{H}(\mathbb{D})$ with square-summable Fourier coefficients and is thus naturally isomorphic to the sequence space $\ell^{2}\left(\mathbb{N}_{0}\right)$. In this setting, the operator $C$ takes the form

$$
\ell^{2}\left(\mathbb{N}_{0}\right) \ni\left(a_{n}\right)_{n \geq 0} \stackrel{C}{\longrightarrow}\left(a_{0}, \frac{a_{0}+a_{1}}{2}, \ldots, \frac{a_{0}+\cdots+a_{n}}{n+1}, \ldots\right),
$$

which Brown, Halmos and Shields termed in 1965 the (discrete) Cesàro operator, [8]. It is they who are credited with beginning the study of this operator from a modern

2000 Mathematics Subject Classification: Primary 47B38, 47A10, 47A11, 47D03.

Key words and phrases: Cesàro operator, operator semigroups, decomposable operators, Bishop's property $(\beta)$.

The paper is in final form and no version of it will be published elsewhere. 
perspective. That the Cesàro operator is bounded on $H^{2}(\mathbb{D})$ is actually due to Hardy, [19], who was interested in simplifying the proof of an inequality by Hilbert. Brown, Halmos and Shields described the spectrum and proved that $\left.C\right|_{H^{2}}$ is hyponormal, i.e., $C^{*} C-C C^{*}$ is a positive operator on the Hilbert space $H^{2}(\mathbb{D})$. In 1971 , Kriete and Trutt [24] extended this result and proved that $\left.C\right|_{H^{2}(\mathbb{D})}$ is subnormal, that is, the restriction of a normal operator to an invariant subspace. In fact, they constructed a measure $\sigma$ on $\overline{\mathbb{D}}$ such that $I-C$ is unitarily equivalent to the multiplication operator $M_{z} f(z)=z f(z)$ on the space $H^{2}(\sigma)$, the closure of the analytic polynomials in $L^{2}(\sigma)$.

The connection of the Cesàro operator to composition operators was first made by Deddens in 1972, who used the result of Kriete and Trutt to show that, for $\psi_{r}(z)=$ $r z+1-r, 0<r \leq 1$, the operator $f \mapsto f \circ \psi_{r}$ is co-subnormal on $H^{2}(\mathbb{D})$, [11]. In fact, the collection $S_{t} \in \mathcal{L}\left(H^{2}(\mathbb{D})\right), S_{t} f=f \circ \psi_{e^{-t}}, t \geq 0$, is a strongly continuous semigroup of composition operators on $H^{2}(\mathbb{D})$. Cowen in $[9]$ was the first to note that the Cesàro operator can also be represented as the resolvent at 0 of the infinitesimal generator of the adjoint semigroup $\left(S_{t}^{*}\right)_{t \geq 0}$ and gave a direct proof of the subnormality of this semigroup. From this, a theorem of Ito, [23, Theorem 4], enabled him to give an alternative proof that $\left.C\right|_{H^{2}(\mathbb{D})}$ is subnormal. See the remarks following Theorem 2.3 below.

The Hilbert space $H^{2}(\mathbb{D})$ is generalized by the Hardy spaces $H^{p}(\mathbb{D}), 1 \leq p<\infty$, consisting of functions $f \in \mathcal{H}(\mathbb{D})$ such that

$$
\|f\|_{H^{p}}^{p}=\sup _{0 \leq r<1} \frac{1}{2 \pi} \int_{0}^{2 \pi}\left|f\left(r e^{i \theta}\right)\right|^{p} d \theta<\infty .
$$

We also consider certain weighted Bergman spaces: let $\mu$ denote normalized planar measure on $\mathbb{D}$, and, for $-1<\alpha$, define $d \mu_{\alpha}(z)=(\alpha+1)\left(1-|z|^{2}\right)^{\alpha} d \mu(z)$. The corresponding weighted Bergman space $L_{a}^{p, \alpha}, 1 \leq p<\infty$, is defined to be the space of all functions $f \in \mathcal{H}(\mathbb{D})$ such that

$$
\|f\|_{p, \alpha}^{p}=\int_{\mathbb{D}}|f(z)|^{p} d \mu_{\alpha}(z)<\infty .
$$

Each $L_{a}^{p, \alpha}$ is a closed subspace of $L^{p}\left(\mu_{\alpha}\right)$ in which the polynomials are dense; see for example [21]. It is not hard to see that the measures $\mu_{\alpha}$ converge weak-* to normalized Lebesgue measure on $\partial \mathbb{D}$ as $\alpha \rightarrow-1^{+}$, and in many ways, the classical Hardy space $H^{p}(\mathbb{D})$ is the limiting case of the weighted Bergman spaces $L_{a}^{p, \alpha}$ as $\alpha \rightarrow-1^{+}$. Allow us to write $L^{p,-1}=H^{p}(\mathbb{D})$, and, for $f \in H^{p}(\mathbb{D})$,

$$
\|f\|_{p,-1}^{p}=\|f\|_{H^{p}}^{p}=\frac{1}{2 \pi} \int_{0}^{2 \pi}\left|f\left(e^{i \theta}\right)\right|^{p} d \theta=\lim _{\alpha \rightarrow-1^{+}}\|f\|_{p, \alpha}^{p},
$$

where, as usual, we identify a function in $H^{p}(\mathbb{D})$ with its boundary values on $\partial \mathbb{D}$.

Siskakis, [35], investigated the semigroups associated with the Cesàro operator in the setting of $H^{p}(\mathbb{D})$, and used estimates on the resolvents to calculate the norm and the spectrum when $2 \leq p<\infty$, and obtained bounds for these in the case $1 \leq p<2$. He then applied similar techniques to study the Cesàro operator on the weighted Bergman spaces $L_{a}^{p, \alpha}, \alpha>-1$, determining the exact norm and spectrum in the case $p \geq 4$ and upper bounds for $1 \leq p<4$, [37], [38]. Using ideas of Hardy and Littlewood found in [20], Miao was able to show that the Cesàro operator is bounded on $H^{p}(\mathbb{D})$ for $0<p<1$, [26]. 
Siskakis's techniques were later exploited in [30] and [27] to obtain a complete spectral picture of the Cesàro operator on the Hardy spaces $H^{p}(\mathbb{D})$, for $p>1$, and on the Bergman spaces $L_{a}^{p, 0}$ for $p>2$. However, due to the existence of eigenvalues, it seems to be impossible to go beyond the limiting case $p=2$ in the Bergman space setting using the methods of composition semigroups. The reason for this is that one needs resolvent estimates around the eigenvalues, while the composition semigroups only give such estimates for larger regions of the spectrum. Dahlner, [10], uses an alternative approach to replicate the spectral results of [30] and [27] for $\left.C\right|_{L_{a}^{p, \alpha}}$, for all $p$ and $\alpha, 1<p,-1<\alpha$.

Very recently, Anna-Maria Persson has shown in her PhD thesis under Alemann's direction that the Cesàro operator is subdecomposable on $H^{1}$ and on the standard weighted Bergman spaces $L_{a}^{1, \alpha}, \alpha \geq 0$. Her method combines some ideas of Dahlner with a technique based on different semigroups of composition operators.

We denote the spectrum of a bounded linear operator $T \in \mathcal{L}(X)$ on a Banach space $X$ by $\sigma(T)$, and its point spectrum and approximate point spectrum by $\sigma_{p}(T)$ and $\sigma_{a p}(T)$, respectively. The left resolvent set for $T$ is the open set $\rho_{\ell}(T)$ consisting of the points $\lambda \in \mathbb{C}$ for which there exists an operator $L \in \mathcal{L}(X)$ such that $L(\lambda-T)=I$. Equivalently, $\lambda \in \rho_{\ell}(T)$ provided $\lambda-T$ is bounded below and the range, $\operatorname{ran}(\lambda-T)$, is complemented in $X$. The left spectrum of $T$ is the set $\sigma_{\ell}(T)=\mathbb{C} \backslash \rho_{\ell}(T) . T$ is said to be a Fredholm operator provided that its null space, $\operatorname{ker}(T)$, is finite dimensional and its range, $\operatorname{ran}(T)$, has finite codimension. This last condition implies that $\operatorname{ran}(T)$ is closed. The index of a Fredholm operator $T$ is defined to be $\operatorname{ind}(T)=\operatorname{dim}(\operatorname{ker}(T))-\operatorname{dim}(X / \operatorname{ran}(T))$. Recall that the function $\lambda \mapsto \operatorname{ind}(\lambda-T)$ is constant on each component of the essential resolvent set $\rho_{e}(T)=\{\lambda \in \mathbb{C}: \lambda-T$ is Fredholm $\}$, an open subset of the complex plane whose complement, $\sigma_{e}(T)$, is the essential spectrum of $T$. See for example Section 3.7 of [25]. It is straightforward to check that $\sigma_{\ell}(T) \subseteq \sigma_{p}(T) \cup \sigma_{e}(T)$ for arbitrary $T \in \mathcal{L}(X)$.

We denote by $V(\lambda, r)$ and $\nabla(\lambda, r)$ respectively the open and closed balls with center $\lambda \in \mathbb{C}$ and radius $r>0$. If $|\lambda|>0$, let $D(\lambda)=V(\lambda,|\lambda|)$. The following theorem summarizes the spectral properties of the Cesàro operator on the spaces $L_{a}^{p, \alpha},[35],[37],[30]$, [27], [28] and [10].

TheOrem 1.1. If $1<p<\infty$ and $-1 \leq \alpha$, then

(1) $\sigma_{p}\left(\left.C\right|_{L_{a}^{p, \alpha}}\right)=\left\{\frac{1}{n}: n \in \mathbb{N}, n<\frac{2+\alpha}{p}\right\}$, and each eigenvalue is simple.

(2) $\sigma\left(\left.C\right|_{L_{a}^{p, \alpha}}\right)=\bar{D}\left(\frac{p}{2(2+\alpha)}\right) \cup \sigma_{p}\left(\left.C\right|_{L_{a}^{p, \alpha}}\right)$.

(3) $\sigma_{a p}\left(\left.C\right|_{L_{a}^{p, \alpha}}\right)=\sigma_{p}\left(\left.C\right|_{L_{a}^{p, \alpha}}\right) \cup \partial D\left(\frac{p}{2(2+\alpha)}\right)$.

(4) $\sigma_{e}\left(\left.C\right|_{L_{a}^{p, \alpha}}\right)=\partial D\left(\frac{p}{2(2+\alpha)}\right)$, moreover, ind $\left(\lambda-\left.C\right|_{L_{a}^{p, \alpha}}\right)=-1$ for all $\lambda \in D\left(\frac{p}{2(2+\alpha)}\right)$.

(5) There is an analytic function $\Lambda: \mathbb{C} \backslash \sigma_{a p}\left(\left.C\right|_{L_{a}^{p, \alpha}}\right) \rightarrow \mathcal{L}\left(L_{a}^{p, \alpha}\right)$ such that $\Lambda(\lambda)(\lambda-C)$ $=I$ for all $\lambda \notin \sigma_{a p}\left(\left.C\right|_{L_{a}^{p, \alpha}}\right)$ and for which there exist constants $a_{1}, a_{2} \geq 0$ and $a$ decreasing function $f:(0, \infty) \rightarrow(0, \infty)$ such that $\int_{0}^{1} \log |f(t)| d t<\infty$, and

$$
\|\Lambda(\lambda)\|_{p, \alpha} \leq a_{1} \exp \left(a_{2}|\lambda|^{-1}\right) f\left(\operatorname{dist}\left(\lambda, \sigma_{a p}\left(\left.C\right|_{L_{a}^{p, \alpha}}\right)\right)\right)
$$

for all $\lambda \notin \sigma_{a p}\left(\left.C\right|_{L_{a}^{p, \alpha}}\right)$.

In the Hardy space case $\alpha=-1$, the analyticity of the left resolvent function $\Lambda$ above is due to Didas, [12, Theorem 4.2.4]. Also note that the case $p=1$ is completely open for 
all $\alpha$. The proof in the Hardy spaces, $p>1$, makes fundamental use of a theorem of Marcel Riesz which implies that the projections $T_{n} f(z)=\sum_{|k|<n} \widehat{f}(k) z^{k}$ are uniformly bounded on each $L^{p}, 1<p<\infty$. It follows that for each $f \in L^{p}$, the trigonometric polynomials $T_{n} f$ converge to $f$ in norm and that $\left(H^{p}\right)^{*}$ is conjugate isomorphic to $H^{q}, 1 / p+1 / q=1$, via $\langle f, g\rangle=\frac{1}{2 \pi} \int_{0}^{2 \pi} f\left(e^{i \theta}\right) \overline{g\left(e^{i \theta}\right)} d \theta,[17$, page 109], but M. Riesz's theorem fails in the $p=1$ case. Moreover, the construction of the left inverse $\Lambda: D\left(\frac{p}{2}\right) \rightarrow \mathcal{L}\left(H^{p}(\mathbb{D})\right)$ makes explicit use of the continuity of the integral operator

$$
A f(z)=\frac{1}{z-1} \int_{1}^{z} f(\omega) d \omega \quad\left(f \in H^{p}(\mathbb{D})\right) .
$$

In fact, $\left.A\right|_{H^{p}(D)}$ is the dual of the Cesàro operator $\left.C\right|_{H^{q}(\mathbb{D})}, 1 / p+1 / q=1$, but $A$ is unbounded on $H^{1}(\mathbb{D})$, see Theorem 2.2 below. Dahlner's proof also breaks down for $p=1$; specifically, Lemma 4.7 of [10] fails for every $L_{a}^{1, \alpha}, \alpha \geq 1$.

2. Semigroups associated with the Cesàro operator. The study of semigroups of composition operators on spaces of analytic functions was initiated by Berkson and Porta, [6]. The resolvent of the generator of such semigroups is typically an averaging integral operator, [36], which we refer to as a generalized Cesàro operator; see Section 4. In this section we recall some basic facts regarding operator semigroups and then identify the semigroups of weighted composition operators investigated by Deddens [11] and Cowen $[9]$ in the $H^{2}(\mathbb{D})$ setting and by Siskakis in the weighted Bergman spaces $L_{a}^{p, \alpha}, 1 \leq p<\infty$, $-1 \leq \alpha,[35],[37]$ and [38]. We indicate some open problems regarding these semigroups in Section 3.

A semigroup of operators on a Banach space $X$ is a net $\left\{S_{t}\right\}_{t \geq 0}$ in $\mathcal{L}(X)$ such that $S_{0}=I$ and $S_{t} S_{s}=S_{t+s}$, whenever $t, s \in[0, \infty)$. The semigroup $\left\{S_{t}\right\}_{t \geq 0}$ is strongly continuous $\left(C_{0}\right)$ provided that, for each $x \in X$, the function $t \rightarrow S_{t} x$ is norm continuous on $[0, \infty)$. In this case, there is a closed, densely defined operator $A$ on $X$ defined by

$$
A x=\lim _{t \rightarrow 0^{+}} \frac{\left(S_{t}-I\right) x}{t}=\left.\frac{\partial S_{t} x}{\partial t}\right|_{t=0},
$$

with domain $\mathcal{D}(A)=\left\{x: \lim _{t \rightarrow 0^{+}} \frac{\left(S_{t}-I\right) x}{t}\right.$ exists $\}$. This operator is always closed, i.e., $A$ has closed graph, and is called the (infinitesimal) generator of the semigroup $\left\{S_{t}\right\}_{t \geq 0}$.

The resolvent set of $A$ is the set of $\lambda \in \mathbb{C}$ for which there exists $R(\lambda, A) \in \mathcal{L}(X)$ such that $R(\lambda, A) X=\mathcal{D}(A), R(\lambda, A)(\lambda-A)=\left.I\right|_{\mathcal{D}(A)}$, and $(\lambda-A) R(\lambda, A)=I_{X}$. We define $\sigma(A)=\mathbb{C} \backslash \rho(A)$, a closed subset of the plane since $A$ is closed. If $\mathbb{C}_{\infty}$ denotes the Riemann sphere, and $A$ is a closed operator on $X$, let $\sigma_{\infty}(A)=\{\infty\} \cup \sigma(A)$ if $A$ is unbounded; otherwise $\sigma_{\infty}(A)=\sigma(A)$.

The type of the semigroup is defined to be

$$
\omega:=\lim _{t \rightarrow \infty} \frac{\log \left\|S_{t}\right\|}{t},
$$

where $-\infty \leq \omega<\infty$. If $\operatorname{Re}(\lambda)>\omega$, then $\lambda \in \rho(A)$ and the Laplace formula holds:

$$
R(\lambda, A) x=\int_{0}^{\infty} e^{-\lambda t} S_{t} x d t
$$


In particular, if $\left\|S_{t}\right\| \leq M e^{\omega t}$ for every $t \geq 0$, then a consequence of the Hille-YosidaPhillips theorem is that $\left\|R(\lambda, A)^{n}\right\| \leq \frac{M}{(\operatorname{Re} \lambda-\omega)^{n}}$ for all $\lambda, \operatorname{Re}(\lambda)>\omega$ and $n \geq 0$. A spectral mapping theorem holds in setting of the Riemann sphere, [14, VII.9.2]: if $\lambda \in \rho(A)$, then $f(z)=(\lambda-z)^{-1}$ is analytic in a neighborhood of $\sigma_{\infty}(A)$ and

$$
\sigma(R(\lambda, A))=f\left(\sigma_{\infty}(A)\right)
$$

The semigroup $\left\{S_{t}\right\}_{t \geq 0}$ is norm continuous if and only if the generator $A$ is bounded, and in this case $S_{t}=e^{A t}$ for all $t \geq 0$. With suitable interpretation, this formula remains true in the strongly continuous case. In particular, a spectral inclusion is valid for $\left(C_{0}\right)$ semigroups, [18, Section 1.9.3]:

$$
\begin{aligned}
\exp \left(t \sigma_{\infty}(A)\right) & \subseteq \sigma\left(T_{t}\right) \text { and } \\
\exp \left(t \sigma_{p}(A)\right) & =\sigma_{p}\left(T_{t}\right) \backslash\{0\}
\end{aligned}
$$

for all $t \geq 0$. Generally, the containment in $(*)$ may be strict since $\sigma(A)$ may in fact be empty. [40, Example 5.4] provides an example that we will return to in Section 3. In the case that equality is obtained for all $t \geq 0$ in $(*)$, we say that the spectral mapping property holds for the semigroup $\left\{S_{t}\right\}_{t \geq 0}$. Standard references for semigroup theory include Chapter VIII of [14], [18], and [22]; we also refer to [34, Chapter 13].

The following theorem follows from Littlewood's subordination theorem and a result of Berkson and Porta [6] in the Hardy space case and is due to Siskakis, [37], for the spaces $L_{a}^{p, \alpha}, \alpha>-1$.

THEOREM 2.1. If $\left\{\psi_{t}\right\}_{t \geq 0}$ is a semigroup of analytic functions $\psi_{t}: \mathbb{D} \rightarrow \mathbb{D}$ such that $(t, z) \mapsto \psi_{t}(z)$ is continuous on $[0, \infty) \times \mathbb{D}$, then, for every $p \geq 1$ and $\alpha \geq-1$, the corresponding composition operators $T_{t} f=f \circ \psi_{t}$ are each continuous on $L_{a}^{p, \alpha}$. Moreover, $\left\{T_{t}\right\}_{t \geq 0}$ is a strongly continuous semigroup in $\mathcal{L}\left(L_{a}^{p, \alpha}\right)$.

A fundamental example is the semigroup of composition operators corresponding to the functions $\psi_{t}(z)=e^{-t} z+1-e^{-t}, t \geq 0$. Notice that 1 is a fixed point for each $\psi_{t}$, and that $\psi_{t} \rightarrow 1$ uniformly on $\mathbb{D}$ as $t \rightarrow \infty$. The spectral properties of a corresponding semigroup of weighted composition operators were obtained by Siskakis in [35] and [37] and are summarized in the following theorem.

THEOREM 2.2. Let $p \geq 1$ and $\alpha \geq-1$ and define $S_{t} \in \mathcal{L}\left(L_{a}^{p, \alpha}\right)$ to be the weighted composition operator $S_{t} f=e^{-t} f \circ \psi_{t}$. Then

(1) $\left\{S_{t}\right\}_{t \geq 0}$ is a strongly continuous semigroup of type $\omega=\lim _{t \rightarrow \infty} \frac{\log \left\|S_{t}\right\|}{t}=-1+\frac{2+\alpha}{p}$.

(2) The generator $\Psi$ of $\left\{S_{t}\right\}_{t \geq 0}$ is given by $\Psi f(z)=(1-z) f^{\prime}(z)-f(z)$ with domain $\mathcal{D}(\Psi)=\left\{f \in L_{a}^{p, \alpha}:(1-z) f^{\prime}(z) \in L_{a}^{p, \alpha}\right\}$

(3) $\sigma(\Psi)=\left\{z: \operatorname{Re}(z) \leq-1+\frac{2+\alpha}{p}\right\}$ and every point of the interior of $\sigma(\Psi)$ is a simple eigenvalue with $\operatorname{ker}(\lambda-\Psi)=\operatorname{span}(1-z)^{-(1+\lambda)}$.

(4) The operator $A f(z)=\frac{1}{1-z} \int_{1}^{z} f(\zeta) d \zeta$ is bounded on $L_{a}^{p, \alpha}$ if and only if $\alpha+2<p$. In this case $A=R(0, \Psi)$, and $A$ has spectrum and point spectrum $\sigma(A)=\bar{D}\left(\frac{p}{2(p-2-\alpha)}\right)$ and $\sigma_{p}(A)=D\left(\frac{p}{2(p-2-\alpha)}\right)$, respectively. 
(5) The spectral mapping property holds for each $S_{t}$ :

$$
\begin{aligned}
\sigma\left(S_{t}\right) & =\left\{\exp (\lambda t): \lambda \in \sigma_{\infty}(\Psi)\right\}=\nabla\left(0, e^{t(-1+(2+\alpha) / p)}\right) \text { and } \\
\sigma_{p}\left(S_{t}\right) & =V\left(0, e^{t(-1+(2+\alpha) / p)}\right) \backslash\{0\} .
\end{aligned}
$$

In the Hardy space case, $1<p<\infty$, the operator $\left.A\right|_{H^{q}(\mathbb{D})}$, where $1 / p+1 / q=1$, is similar to the adjoint of the Cesàro operator $\left.C\right|_{H^{p}(\mathbb{D})}$. In the unweighted Bergman space setting, Theorem 2.2 implies that $\left.A\right|_{L_{a}^{p, 0}}$ is bounded only if $p>2$, and in this case, a computation, [27], shows that

$$
A J=J\left(\left.C\right|_{L_{a}^{p /(p-1), 0}}\right)^{*},
$$

where $J$ is the Voltera operator $J f(z)=\frac{1}{z} \int_{0}^{z} f(\zeta) d \zeta$, which is compact on every $L_{a}^{p, \alpha}, p \geq$ $1, \alpha \geq-1$ by [4] and [5].

The semigroup first studied by Cowen is

where

$$
T_{t} f(z)=\frac{\varphi_{t}(z)}{z} f\left(\varphi_{t}(z)\right)
$$

$$
\varphi_{t}(z)=\frac{e^{-t} z}{\left(e^{-t}-1\right) z+1},
$$

for all $t \geq 0$. $\left\{\varphi_{t}\right\}_{t \geq 0}$ is a semigroup of analytic functions $\varphi_{t}: \overline{\mathbb{D}} \rightarrow \overline{\mathbb{D}}$ satisfying the hypotheses of Theorem 2.1 and with common fixed points 0 and 1 . Moreover, for each $t \geq 0, \varphi_{t}(\mathbb{D})$ is a disk internally tangent to $\partial \mathbb{D}$ at $1, \varphi_{t}(\mathbb{D}) \rightarrow D\left(\frac{1}{2}\right)$ as $t \rightarrow \infty$, and the function $z \mapsto \frac{\varphi_{t}(z)}{z}$ is bounded with positive real part. The strong continuity of the semigroup $\left\{T_{t}\right\}_{t \geq 0} \subset \mathcal{L}\left(L_{a}^{p, \alpha}\right)$ follows from Theorem 2.1; and the generator was shown in [35] and [37] to be

$$
\Phi f(z)=(z-1)(z f(z))^{\prime}
$$

with domain $\mathcal{D}(\Phi)=\left\{f \in L_{a}^{p, \alpha}:(z-1)(z f(z))^{\prime} \in L_{a}^{p, \alpha}\right\}, p \geq 1$ and $\alpha \geq-1$. Thus $\left.C\right|_{L_{a}^{p, \alpha}}=R(0, \Phi)$, and so Theorem 1.1 gives us the spectrum and point spectrum of $\Phi$. For the cases $\alpha=-1$ and $\alpha=0$, sharper estimates of $\left\|T_{t}\right\|_{L_{a}^{p, \alpha}}$ exist for $p \geq 2(2+\alpha)$, [35], [37], and we can identify the spectrum of $T_{t}$ in these cases.

Theorem 2.3. Let $p>1$ and $\alpha \geq-1$. Then

(1) $\Phi$ on $L_{a}^{p, \alpha}$ has spectrum the half plane $\sigma\left(\left.\Phi\right|_{L_{a}^{p, \alpha}}\right)=\left\{z: \operatorname{Re}(z) \leq-\frac{2+\alpha}{p}\right\}$ and point spectrum $\sigma_{p}\left(\left.\Phi\right|_{L_{a}^{p, \alpha}}\right)=\left\{-n: n \in \mathbb{N}, n<\frac{2+\alpha}{p}\right\}$. Moreover, each eigenvalue is simple.

(2) $\sigma\left(\left.T_{t}\right|_{\left.L_{a}^{p, \alpha}\right)} \supseteq \nabla\left(0, e^{-t(2+\alpha) / p}\right)\right.$ for all $t \geq 0$.

(3) For $\alpha=-1,0$ and $p \geq 2(2+\alpha),\left\|T_{t}\right\|_{L_{a}^{p, \alpha}}=e^{-t(2+\alpha) / p}$ and the spectral mapping property holds:

$$
\sigma\left(\left.T_{t}\right|_{L_{a}^{p, \alpha}}\right)=\exp \left(t \sigma_{\infty}\left(\left.\Phi\right|_{L_{a}^{p, \alpha}}\right)\right)
$$

If $H$ is a Hilbert space and if $\left\{S_{t}\right\}_{t \geq 0}$ is a strongly continuous semigroup of subnormal operators on $H$, then there is a Hilbert space $K \supset H$ and a strongly continuous semigroup of normal operators $\left\{T_{t}\right\}_{t \geq 0} \subset \mathcal{L}(K)$ such that $\left.T_{t}\right|_{H}=S_{t}$ for each $t$, [23, Theorem 4]. It follows by a classical result of M. H. Stone, [34, Theorem 13.38], that the generator $N$ of $\left\{T_{t}\right\}_{t \geq 0}$ is normal; consequently, the generator $A$ of $\left\{S_{t}\right\}_{t \geq 0}$ is subnormal, and 
each resolvent $R(\lambda, A) \in \mathcal{L}(H)$ is subnormal as well. In particular, subnormality of the semigroup $\left\{T_{t}\right\}_{t \geq 0} \subset H^{2}(\mathbb{D})$ in the previous theorem implies that the Cesàro operator is subnormal, see [9].

Conversely, if $A$ is subnormal and generates a $\left(C_{0}\right)$ semigroup $\left\{S_{t}\right\}_{t \geq 0}$, then $R(\lambda, A)$ is subnormal for each $\lambda \in \rho(A)$, and thus the proof of the Hille-Yosida-Phillips Theorem, [14, VIII.1.13], together with the fact that the set of subnormal operators is SOT-closed in $\mathcal{L}(H)$, [7], implies that each $S_{t}$ is itself subnormal. In particular, the subnormality of the Cesàro operator on $H^{2}(\mathbb{D})$ implies that, for $\psi_{t}(z)=e^{-t} z+1-e^{-t}$, the composition operators $f \mapsto f \circ \psi_{t}$ are co-subnormal on $H^{2}(\mathbb{D})$, a result originally obtained by Deddens, [11].

3. Subdecomposable operators. In attempts to extend the spectral theory of normal operators to the setting of Banach spaces, a variety of classes of operators have been investigated since the 1950s. An operator $T \in \mathcal{L}(X)$ is decomposable in the sense of Foiaş if, for any open cover $\left\{U_{1}, U_{2}, \ldots, U_{n}\right\}$ of the spectrum, $\sigma(T)$, there exist closed, $T$-invariant subspaces $X_{1}, X_{2}, \ldots, X_{n} \subset X$ so that $X=X_{1}+X_{2}+\cdots X_{n}$ and $\sigma\left(\left.T\right|_{X_{j}}\right) \subset U_{j}$ for each $j, 1 \leq j \leq n$, [25], [39]. While decomposable operators typically have no functional calculus beyond the Riesz analytic functional calculus, many of the spectral properties of normal operators in fact hold for decomposable operators. Besides normal operators, or more generally, operators with a rich functional calculus, the class of decomposable operators contains all operators with totally disconnected spectrum; specifically, compact Banach space operators are decomposable.

An operator is said to be subdecomposable provided that it is the restriction of a decomposable operator to a closed invariant subspace. Examples of subdecomposable operators include hyponormal operators on Hilbert spaces, [33] and Banach space isometries, $[25,5.1 .4]$ and [13]. If decomposability is the appropriate generalization of normal operators to Banach spaces, the relation between subnormal and subdecomposable operators is perhaps even stronger. Several results first established for subnormal operators have been shown to hold for subdecomposable operators. As an especially striking example, Eschmeier and Prunaru applied the Scott Brown technique, originally used to show the existence of invariant subspaces for subnormal operators, to prove that subdecomposable operators with thick spectra have invariant subspaces, [16].

Moreover, just as for subnormality, there is an intrinsic characterization of subdecomposability. Let $\mathcal{H}(U, X)$ denote the Fréchet space of analytic, $X$-valued functions defined on an open subset $U \subset \mathbb{C}$, again with the topology of uniform convergence on compact subsets. Every operator $T \in L(X)$ induces a continuous mapping $T_{U}$ on $\mathcal{H}(U, X)$ given by $\left(T_{U} f\right)(\lambda)=(\lambda-T) f(\lambda)$ for all $f \in \mathcal{H}(U, X)$ and $\lambda \in U$. The operator $T \in \mathcal{L}(X)$ is said to have Bishop's property $(\beta)$, [7], provided that for every open $U \subset \mathbb{C}$, the mapping $T_{U}$ is injective and has closed range. Thus, $T$ has property $(\beta)$ if, whenever $\left(f_{n}\right)_{n}$ is a sequence of analytic $X$-valued functions such that $(\lambda-T) f_{n}(\lambda) \rightarrow 0$ uniformly on the compact subsets of an open $U \subseteq \mathbb{C}$, then $f_{n}(\lambda) \rightarrow 0$ uniformly on the compact subsets of $U$. This seemingly technical property in fact completely characterizes the restrictions of bounded decomposable operators to invariant subspaces, and $T \in \mathcal{L}(X)$ is decomposable if and only if both $T$ and $T^{*}$ have property $(\beta),[1],[25]$. 
The notions of decomposability and property $(\beta)$ extend to the setting of $\mathcal{C}_{d}(X)$, the space of closed, densely defined operators on a Banach space $X$. An operator $A \in \mathcal{C}_{d}(X)$ is said to have the Spectral Decomposition property (SDP) provided that, whenever $U_{0}, U_{1}, \ldots U_{n}$ is an open cover of $\sigma_{\infty}(A)$ such that $U_{j}$ is bounded for every $j, 1 \leq j \leq n$, there exist closed, $A$-invariant subspaces $X_{0}, X_{1}, \ldots, X_{n}$ such that $X=X_{0}+X_{1}+\cdots+X_{n}$ and $\sigma_{\infty}\left(\left.A\right|_{X_{j}}\right) \subset U_{j}$ for each $j \geq 0$. Note that in the terminology of Vasilescu, an operator with the $\mathrm{SDP}$ is referred to as being $\{\infty\}$-decomposable, [39, IV.4.12]. Clearly, in the case of bounded operators, SDP is equivalent to decomposability. Moreover, by [39, IV.4.16], every $A \in$ $\mathcal{C}_{d}(X)$ satisfies a version of property $(\beta)$ : if $U$ is an open subset $\mathbb{C}$ and $\left(f_{n}\right)_{n \geq 1}$ is a sequence of analytic functions in $\mathcal{D}\left(A_{U}\right)$ such that $A_{U} f_{n} \rightarrow 0$ in $\mathcal{H}(U, X)$, then $f_{n} \rightarrow 0 \in \mathcal{H}(U, X)$.

Just as in the bounded case, if $A \in \mathcal{C}_{d}(X)$ and $U$ is an open subset of $\mathbb{C}$, we define $A_{U}$ on $\mathcal{H}(U, X)$ by $A_{U} f(\lambda)=(\lambda-A) f(\lambda)$, with domain $\mathcal{D}\left(A_{U}\right)=\{f \in \mathcal{H}(U, X): A f \in$ $H(U, X)\}$. It is easy to see that $A$ closed implies that $A_{U}$ is a closed operator on $\mathcal{H}(U, X)$; moreover, the fact that $\mathcal{H}(U, X)$ is isomorphic to the completion of the projective tensor product of $\mathcal{H}(U)$ and $X,\left[25\right.$, A.3.5], implies that $A_{U}$ is densely defined on $\mathcal{H}(U, X)$ whenever $\mathcal{D}(A)$ is dense in $X$. Also notice that by the closed graph theorem, [34, 2.15], a closed Fréchet space mapping is injective and with closed range precisely when it is the inverse of a continuous map.

Proposition 3.1. Suppose that $A \in \mathcal{C}_{d}(X)$ and that $U$ and $V$ are open subsets of $\mathbb{C}$. If both $A_{U}$ and $A_{V}$ are injective with closed range, then $A_{U \cup V}$ is injective and has closed range as well.

Proof. Without loss of generality, we assume that $U \cap V \neq \emptyset$. Define continuous mappings $r: \mathcal{H}(U \cup V, X) \rightarrow \mathcal{H}(U, X) \oplus \mathcal{H}(V, X)$ and $q: \mathcal{H}(U, X) \oplus \mathcal{H}(V, X) \rightarrow \mathcal{H}(U \cap V, X)$ by $r f=\left(\left.f\right|_{U},\left.f\right|_{V}\right)$ and $q(f, g)=\left.(f-g)\right|_{U \cap V}$. Then the sequence

$$
0 \rightarrow \mathcal{H}(U \cup V, X) \stackrel{r}{\rightarrow} \mathcal{H}(U, X) \oplus \mathcal{H}(V, X) \stackrel{q}{\rightarrow} \mathcal{H}(U \cap V, X) \rightarrow 0
$$

is exact by [25, 2.1.7]; in particular, $r$ is injective with closed range. Moreover, $r\left(\mathcal{D}\left(A_{U \cup V}\right)\right)$ $=\mathcal{D}\left(A_{U} \oplus A_{V}\right) \cap \operatorname{ran}(r)$, and $r A_{U \cup V}=\left(A_{U} \oplus A_{V}\right) r$. Clearly, $A_{U \cup V}$ is injective if both $A_{U}$ and $A_{V}$ are injective. Thus, we need only show that $\operatorname{ran}\left(A_{U \cup V}\right)$ is closed, and since $r$ is injective with closed range, this is equivalent to showing $\operatorname{ran}\left(r A_{U \cup V}\right)$ is closed. If $\left(f_{n}\right)_{n \geq 1} \subset \mathcal{D}\left(A_{U \cup V}\right)$ is such that $\left(A_{U} \oplus A_{V}\right) r f_{n} \rightarrow\left(g_{1}, g_{2}\right) \in \mathcal{H}(U, X) \oplus \mathcal{H}(V, X)$, then the assumption that $A_{U}$ and $A_{V}$ are closed, injective mappings with closed ranges implies that $\left(r f_{n}\right)_{n \geq 1}$ converges to an $\left(h_{1}, h_{2}\right) \in \mathcal{D}\left(A_{U} \oplus A_{V}\right)$ such that $\left(A_{U} \oplus A_{V}\right)\left(h_{1}, h_{2}\right)=\left(g_{1}, g_{2}\right)$. But, in this case, $\left(h_{1}, h_{2}\right) \in \operatorname{ran}(r) \cap \mathcal{D}\left(A_{U} \oplus A_{V}\right)=r\left(\mathcal{D}\left(A_{U \cup V}\right)\right)$, and so $\operatorname{ran}\left(r A_{U \cup V}\right)=$ $\left(A_{U} \oplus A_{V}\right) r\left(\mathcal{D}\left(A_{U \cup V}\right)\right)$ is closed, as desired.

Theorem 3.2. Suppose that $A \in \mathcal{C}_{d}(X)$ and that $U$ is an open subset of $\mathbb{C}$.

(1) $A_{U}$ is injective with closed range in $\mathcal{H}(U, X)$ if and only if $f_{n} \rightarrow 0$ in $\mathcal{H}(U, X)$ whenever $\left(f_{n}\right)_{n \geq 1} \subset \mathcal{D}\left(A_{U}\right)$ such that $A_{U} f_{n} \rightarrow 0$. In particular, $A$ has Bishop's property $(\beta)$ if and only if $A_{U}$ is injective with closed range for every open $U \subset \mathbb{C}$.

(2) If $\lambda \in \rho(A)$, then $A$ has property $(\beta)$ if and only if the resolvent $R(\lambda, A)$ has property $(\beta)$.

(3) A has the SDP if and only if both $A$ and $A^{*}$ have Bishop's property $(\beta)$. 
Proof. The third statement is due to I. Erdelyi and S. Wang, [15]. We sketch a proof of (1) and (2) for the convenience of the reader. If $A$ has property $(\beta)$ and $\left(f_{n}\right)_{n} \subset \mathcal{D}\left(A_{U}\right)$ is such that $A_{U} f_{n} \rightarrow g$ in $\mathcal{H}(U, X)$, then property $(\beta)$ implies that $\left(f_{n}\right)_{n}$ is a Cauchy sequence. Indeed, if $d$ is a metric on $\mathcal{H}(U, X)$ compatible with the Fréchet topology, then $\left(f_{n}\right)_{n}$ non-Cauchy implies that there is a $\delta>0$ and a subsequence $\left(f_{n_{k}}\right)_{k}$ so that $d\left(f_{n_{k}}, f_{n_{k+1}}\right) \geq \delta$ for all $k$. But, in this case, since $A_{U}\left(f_{n_{k}}-f_{n_{k+1}}\right) \rightarrow 0$, property $(\beta)$ implies that $f_{n_{k}}-f_{n_{k+1}} \rightarrow 0$, a contradiction. Now, if $f_{n} \rightarrow f$, then $\left(f_{n}, A_{U} f_{n}\right) \rightarrow(f, g)$ in $\mathcal{H}(U, X) \times \mathcal{H}(U, X)$; Since $A_{U}$ is a closed operator, it follows that $f \in \mathcal{D}\left(A_{U}\right)$ and $A_{U} f=g$. The converse follows from the closed graph theorem.

To prove (2), we may assume that $\lambda=0$; write $R=R(0, A)$ and let $U \subset \mathbb{C}$ be open. We consider the cases $0 \notin U$ and $0 \in U$. In the first case, define $h(z)=-1 / z$ and let $V=h(U)$. Then $V$ is an open subset of $\mathbb{C} \backslash\{0\}$ and the composition operator $C_{h} f=f \circ h$ is an isomorphism between $\mathcal{H}(U, X)$ and $\mathcal{H}(V, X)$. Let $\mu=h(\lambda)$ for $\lambda \in U$. Then, for $f, g \in \mathcal{H}(U, X)$,

$$
\begin{aligned}
R_{U} f=g & \Leftrightarrow(\lambda-R) f(\lambda)=g(\lambda) \quad(\lambda \in U) \\
& \Leftrightarrow(-\lambda A-I) R f(\lambda)=g(\lambda) \quad(\lambda \in U) \\
& \Leftrightarrow(\mu-A)(h(\mu) R f \circ h(\mu))=g \circ(\mu) \quad(\mu \in V) .
\end{aligned}
$$

Thus $g \circ h \in \operatorname{ran} A_{V}$ whenever $g \in R_{U} \mathcal{H}(U, X)$. Conversely, if $g \circ h=A_{V} h$ for some $f \in \mathcal{D}\left(A_{V}\right)$ then, with $k=-A f$ and $\lambda=h(\mu)$,

$$
g(\lambda)=g \circ h(\mu)=(\mu-A) R k(\mu)=(\lambda-R)(-\lambda(k \circ h)(\lambda)) ;
$$

thus $g \in \operatorname{ran} R_{U}$ and we have that $\operatorname{ran} A_{V}=C_{h}$ ran $R_{U}$; in particular, ran $A_{V}$ is closed if and only if ran $R_{U}$ is closed. Similarly, $A_{V}$ is injective if and only if $R_{U}$ is injective, and therefore, $A_{U}$ is injective and with closed range for every open subset $U \subseteq \mathbb{C} \backslash\{0\}$ if and only if $R_{U}$ is injective and with closed range for every $U \subseteq \mathbb{C} \backslash\{0\}$.

Now, consider $U$ open with $0 \in U$ and suppose that $A_{U}$ has property $(\beta)$ and that $R_{U} f_{n} \rightarrow 0$ in $\mathcal{H}(U, X)$. Fix $K$ compact in $U$ and let $\epsilon>0$ be such that $V(0,2 \epsilon) \subset U$. If $G=V(0,2 \epsilon) \backslash \nabla(0,3 / 2 \epsilon)$, then $R_{G} f_{n} \rightarrow 0$ and the argument above implies that $f_{n} \rightarrow 0$ in $\mathcal{H}(G, X)$. By Cauchy's formula, it follows that $f_{n} \rightarrow 0$ uniformly on $\nabla(0, \epsilon)$. If $H=U \backslash \nabla(0, \epsilon / 2)$, then the argument of the last paragraph again implies that $R_{H}$ is injective with closed range. Since $R_{H} f_{n} \rightarrow 0, f_{n} \rightarrow 0$ uniformly on $K \backslash V(0, \epsilon) \subset H$. Therefore $f_{n} \rightarrow 0$ uniformly on $K$, and, since $K$ was an arbitrary compact subset of $U$, it follows that $f_{n} \rightarrow 0$ in $\mathcal{H}(U, X)$. Thus $R$ has property $(\beta)$ whenever $A$ does. The other implication follows by a similar argument.

Growth conditions on the resolvent of an operator have long been recognized as an important tool in spectral theory, and log-log integrability criteria provide sufficient conditions for Bishop's property $(\beta)$. Here is a version relevant to our setting; see [25, 1.7.4].

TheOrem 3.3. Suppose that $T \in \mathcal{L}(X)$ with $\sigma(T)$ a closed disk. Let $V$ be an open neighborhood of $\sigma(T)$, and suppose there exists a set $\mathcal{F}$ that accumulates only on a compact, totally disconnected subset $E$ of $\partial \sigma(T)$, a locally bounded function $\omega: V \backslash \overline{\mathcal{F}} \rightarrow(0, \infty)$, and a decreasing function $f:(0, \infty) \rightarrow(0, \infty)$ such that 


$$
\int_{0}^{1} \log ^{+} \log ^{+} f(t) d t<\infty \quad \text { and } \quad\|x\| \leq \omega(\lambda) f(\operatorname{dist}(\lambda, \partial \sigma(T))\|(\lambda-T) x\|
$$

for all $x \in X$ and $\lambda \in V \backslash(\mathcal{F} \cup \partial \sigma(T))$. Then $T$ has Bishop's property $(\beta)$.

In conjunction with Theorem 1.1, this immediately implies the following.

Theorem 3.4. For every $p>1$ and $\alpha \geq-1$, the Cesàro operator $C \in \mathcal{L}\left(L_{a}^{p, \alpha}\right)$ has Bishop's property $(\beta)$.

Since $C$ is the resolvent $R(0, \Phi)$ of $\Phi f(z)=(z-1)(z f(z))^{\prime}$, it therefore follows from Theorem 3.2 that $\Phi \in \mathcal{C}_{d}\left(L_{a}^{p, \alpha}\right)$ has Bishop's property $(\beta)$ as well, $p>1, \alpha \geq-1$.

In light of the results for subnormal semigroups, it is natural to investigate conditions under which subdecomposability of a semigroup implies that of its generator, and conversely. The uniformly continuous case is straightforward: $\left\{S_{t}\right\}_{t \geq} \subset \mathcal{L}(X)$ is a uniformly continuous semigroup if and only if its generator, $A$, is bounded, and in this case, $S_{t}=\exp (A t)$ for each $t$. It therefore follows from [25, 3.3.9] that $A$ is subdecomposable if and only if $S_{t_{0}}$ is subdecomposable for some $t_{0}>0$, equivalently, if $S_{t}$ is subdecomposable for all $t>0$. Shengwang Wang has shown that at least one implication holds more generally, [40].

THEOREM 3.5. Let $X$ be a complex Banach space and suppose that $\left\{T_{t}\right\}_{t \geq 0}$ is a $\left(C_{0}\right)$ semigroup in $\mathcal{L}(X)$ with generator $A$. If $T_{t_{0}}$, respectively $T_{t_{0}}^{*}$, has Bishop's property $(\beta)$ for some $t_{0}>0$ then $A$, respectively $A^{*}$, does as well. In particular, $A$ is $\{\infty\}$-decomposable provided $T_{t_{0}}$ is decomposable for some $t_{0}>0$.

[40, Example 5.4] provides an example of a $\left(C_{0}\right)$ semigroup $\left\{T_{t}\right\}_{t \geq 0}$ such that $T_{t}$ fails to be decomposable for each $t>0$ but for which the generator has empty spectrum and therefore trivially the SDP. Notice that the spectral mapping property fails spectacularly in this example. Also, recall that the proof that subnormality of the generator of a $\left(C_{0}\right)$ semigroup $\left\{T_{t}\right\}_{t \geq 0}$ implies that each $T_{t}$ is subnormal makes use of the fact that the set of subnormal operators is SOT-closed in $\mathcal{L}(H)$, while the set of subnormal, and thus subdecomposable operators, is SOT-dense, [32]. Thus some stronger regularity assumption of $\left\{T_{t}\right\}_{t \geq 0}$ seems to be necessary in order to obtain a converse of Wang's theorem.

The work of Deddens and Cowen concerning subnormality of the semigroup $\left\{T_{t}\right\}_{t \geq 0} \subset$ $\mathcal{L}\left(H^{2}(\mathbb{D})\right)$, where $T_{t} f(z)=\frac{\varphi_{t}(z)}{z} f\left(\varphi_{t}(z)\right)$, suggests the question of whether these operators are subdecomposable on the Hardy spaces $H^{p}(\mathbb{D}), p \neq 2$, or, more generally, on $L_{a}^{p, \alpha}$, $p>1, \alpha \geq-1$. Indeed, this was the impetus for the original work establishing property $(\beta)$ for the Cesàro operator. But subdecomposability of the operators $T_{t}$ remains open in every case. Since the spectral mapping property holds for $\left\{T_{t}\right\}_{t \geq 0} \subset \mathcal{L}\left(L_{a}^{p, \alpha}\right)$, perhaps a converse of Wang's theorem can be shown to hold in this special case.

4. Generalized Cesàro operators. Given $g \in \mathcal{H}(\mathbb{D})$, we define the corresponding generalized Cesàro operator $S_{g}: \mathcal{H}(\mathbb{D}) \rightarrow \mathcal{H}(\mathbb{D})$ by

$$
S_{g} f(z)=\frac{1}{z} \int_{0}^{z} f(\omega) g(\omega) d \omega
$$

for all $z \in \mathbb{D}$. Since $\mathcal{H}(\mathbb{D})$ is a Fréchet algebra, each $S_{g}$ is continuous on $\mathcal{H}(\mathbb{D})$. 
In [36], Siskakis considered a class of generalized Cesàro operators associated with semigroups of weighted composition operators on $H^{p}(\mathbb{D}), 1 \leq p<\infty$. Specifically, $S_{g}$ is the resolvent of the generator of a $\left(C_{0}\right)$ semigroup of weighted composition operators $T_{t} f(z)=\frac{\phi_{t}(z)}{z} f\left(\phi_{t}(z)\right)$ for some continuous semigroup of functions $\phi_{t}: \mathbb{D} \rightarrow \mathbb{D}$ with $\phi_{t}(0)=0$, if and only if $\operatorname{Re}(g(z))>0$ for all $z \in \mathbb{D}$. In this case, $S_{g}$ is bounded on each $H^{p}(\mathbb{D}), 1 \leq p$, and compactness of $S_{g}$ is characterized in terms of the Herglotz representation of $g$. In the process, Siskakis identified the spectrum of the operator $\left.S_{g}\right|_{H^{p}}$ for the particular case $g(z)=(1+z) /(1-z)$.

For general $g \in \mathcal{H}(\mathbb{D})$, continuity of $S_{g}$ on $L_{a}^{p, \alpha}$ is characterized in terms of functiontheoretic properties of $g$. If $G(z)=\int_{0}^{z} g(w) d w$, then Pommerenke [31] showed that $S_{g}$ is bounded on the Hilbert space $H^{2}(\mathbb{D})$ if and only if $G \in$ BMOA. Aleman and Siskakis [4] extended Pommerenke's result to the Hardy spaces $H^{p}(\mathbb{D})$ for all $p, 1 \leq p<\infty$, and showed that $S_{g}$ is compact on $H^{p}(\mathbb{D})$ if and only if $G \in \mathrm{VMOA}$. The case $0<p<1$ was subsequently settled by Aleman and Cima [3]. In the weighted Bergman spaces $L_{a}^{p, \alpha}$, $\alpha>-1$ and $p \geq 1$, Aleman and Siskakis [5] have shown that $S_{g}$ is bounded, respectively, compact if and only if the primitive $G$ is in the Bloch, resp. little Bloch, space.

In [41] and [42], Young began the study of spectral properties of generalized Cesàro operators on the Hardy space $H^{2}(\mathbb{D})$ and the unweighted Bergman space $L_{a}^{2,0}$, and his results have recently been extended to the weighted Bergman spaces $L_{a}^{p, \alpha}, \alpha \geq-1$ and $1<p<\infty$, [2], from which the following is derived.

We consider the operators $S_{g}$ on $L_{a}^{p, \alpha}, p \geq 1$ and $\alpha \geq-1$, corresponding to functions $g=q+h$, where $q$ is rational and $h \in H^{\infty}(\mathbb{D})$, the space of bounded analytic functions on $\mathbb{D}$. In order for $S_{g}$ to be bounded on $L_{a}^{p, \alpha}$, it is necessary that $q$ be analytic on $\mathbb{D}$ and that all of the poles of $q$ on $\partial \mathbb{D}$ be simple. Thus we may write

$$
g(z)=\sum_{j=1}^{m} \frac{a_{j}}{1-b_{j} z}+h(z),
$$

where $h \in H^{\infty}(\mathbb{D}),\left|b_{j}\right|=1$ for each $j, 1 \leq j \leq m, b_{j} \neq b_{k}$ if $j \neq k$ and where each $a_{j} \neq 0$. Thus

$$
S_{g}=\sum_{j=1}^{m} a_{j} S_{1 /\left(1-b_{j} z\right)}+S_{h} .
$$

Notice that if $|b|=1$, then the composition operator $U_{b} f(z)=f(b z)$ is a surjective isometry on each $L_{a}^{p, \alpha}, p \geq 1$ and $\alpha \geq-1$. Since $S_{1 /(1-b z)}=U_{b} C U_{\bar{b}}$, it follows that $S_{1 /(1-b z)}$ and $C$ have equal spectra, (approximate) point spectra and essential spectra. The key to determining the spectral picture of such operators is the following, [2], together with Theorem 1.1.

LEMMA 4.1. Let $h$ be an absolutely continuous function on the interval $[0,1]$ and define $K_{h}$ on $\mathcal{H}(\mathbb{D})$ by

$$
K_{h} f(z)=\int_{0}^{1} f(t z) h(t) d t \quad(z \in \mathbb{D}) .
$$

Then $K_{h}: L_{a}^{p, \alpha} \rightarrow L_{a}^{p, \alpha}$ is compact for all $p \geq 1$ and $\alpha \geq-1$. In particular, if $\varphi \in H^{\infty}(\mathbb{D})$, then $S_{\varphi}$ is compact on each $L_{a}^{p, \alpha}$. Moreover, if $b_{1}$ and $b_{2}$ are distinct points on $\partial \mathbb{D}$, then the product $S_{1 /\left(1-b_{1} z\right)} S_{1 /\left(1-b_{2} z\right)}$ is compact. 
Let $\mathcal{X}_{S}$ denote the characteristic function of a subset $S$ and recall our notation $D(\lambda)=$ $V(\lambda,|\lambda|)$ for all $\lambda \neq 0$.

THEOREM 4.2. Suppose that $g(z)=\sum_{j=1}^{m} \frac{a_{j}}{1-b_{j} z}+h(z)$ where $b_{j}, 1 \leq j \leq m$, are distinct points on the unit circle $\partial \mathbb{D},\left|a_{j}\right|>0$ for each $j$, and $h \in H^{\infty}(\mathbb{D})$. Then for every $\alpha \geq-1$ and $p>1$,

(1) $S_{g} \in \mathcal{L}\left(L_{a}^{p, \alpha}\right)$ has point spectrum

$$
\sigma_{p}\left(\left.S_{g}\right|_{L_{a}^{p, \alpha}}\right)=\left\{\frac{g(0)}{n}: \Re\left(\frac{a_{j}}{g(0)}\right)<\frac{2+\alpha}{n p}, 1 \leq j \leq m\right\},
$$

each eigenvalue of $S_{g}$ is simple, and if $h(0)=0$, then $\sigma_{p}\left(\left.S_{g}\right|_{L_{a}^{p, \alpha}}\right)$ is finite.

(2) $\sigma\left(\left.S_{g}\right|_{L_{a}^{p, \alpha}}\right)=\sigma_{p}\left(\left.S_{g}\right|_{L_{a}^{p, \alpha}}\right) \cup \bigcup_{j=1}^{m} \bar{D}_{j}$, where $D_{j}=D\left(\frac{p a_{j}}{2(2+\alpha)}\right)$, for each $j, 1 \leq j \leq m$.

(3) $S_{g} \in \mathcal{L}\left(L_{a}^{p, \alpha}\right)$ has essential spectrum $\sigma_{e}\left(S_{g}\right)=\bigcup_{j=1}^{m} \partial D_{j}$. Moreover, if $\lambda \in \rho_{e}\left(S_{\varphi}\right)$, then ind $\left(\lambda-S_{g}\right)=-\sum_{j=1}^{m} \mathcal{X}_{D_{j}}(\lambda)$.

(4) The left spectrum is $\sigma_{\ell}\left(\left.S_{g}\right|_{L_{a}^{p, \alpha}}\right) \subseteq \sigma_{p}\left(\left.S_{g}\right|_{L_{a}^{p, \alpha}}\right) \cup \bigcup_{j=1}^{m} \partial D_{j}$.

In [36], Siskakis raised the question of determining subnormality or hyponormality of the operators $S_{g} \in H^{2}(\mathbb{D})$. Subnormality of the operators $S_{a /(1-b z)} \in \mathcal{L}\left(H^{2}(\mathbb{D}),|b|=1\right.$, $a \neq 0$, follows trivially from the fact that the Cesàro operator is subnormal on $H^{2}(D)$; no other examples are known, and these in fact were the only known examples of hyponormal generalized Cesàro operators until it was shown in [2] that $S_{(1+z) /(1-z)}$ is hyponormal on $H^{2}(\mathbb{D})$. This was done by showing that the principal minors of the matrix for $S_{(1+z) /(1-z)}^{*} S_{(1+z) /(1-z)}-S_{(1+z) /(1-z)} S_{(1+z) /(1-z)}^{*}$ corresponding to the canonical basis for $H^{2}(\mathbb{D})$ are all positive definite, and this method also applies in the unweighted Bergman space $L_{a}^{2,0}$. It is well known that if $T \in \mathcal{L}(H)$ is hyponormal, then $\|T\|=r(T)$, the spectral radius of $T$. Since, by Theorem 1.1, $r\left(\left.C\right|_{L_{a}^{2,0}}\right)=1$ while $C 1(z)=\sum_{0}^{\infty} \frac{1}{n+1} z^{n}$ has norm $\|C 1\|_{2,0}^{2}=\sum_{n=0}^{\infty} \frac{1}{(n+1)^{2}}>1$, it follows that $C \in \mathcal{L}\left(L_{a}^{2,0}\right)$ is not hyponormal. A similar argument applies to the operators $S_{1 /\left(1-z^{n}\right)}, n \geq 2$, on each $L_{a}^{2, \alpha}, \alpha \geq-1$, [2]. Indeed, if $p$ is a polynomial of degree $n \geq 2$ with distinct zeros $\zeta_{1}, \zeta_{2}, \ldots, \zeta_{n}$, then the partial fraction decomposition of $1 / p$ is

$$
\frac{1}{p(z)}=\sum_{k=1}^{n} \frac{1}{p^{\prime}\left(\zeta_{k}\right)\left(z-\zeta_{k}\right)}
$$

and therefore,

$$
\frac{1}{1-z^{n}}=\frac{1}{n} \sum_{k=0}^{n-1} \frac{1}{1-e^{-2 k \pi i / n} z} .
$$

By Theorem 4.2, $\left.S_{1 /\left(1-z^{n}\right)}\right|_{L_{a}^{2, \alpha}}$ has spectrum $\bar{D}\left(\frac{1}{n(2+\alpha)}\right) \cup\left\{\frac{1}{k}: k<\frac{(2+\alpha) n}{2}\right\}$ and thus spectral radius at most 1 . On the other hand,

$$
\left\|S_{1 /\left(1-z^{n}\right)} 1\right\|_{2, \alpha}^{2}=\sum_{k=0}^{\infty} \frac{1}{(1+n k)^{2}}\left\|z^{n k}\right\|_{2, \alpha}^{2}>1,
$$

and therefore $S_{1 /\left(1-z^{n}\right)}$ fails to be hyponormal on $L_{a}^{2, \alpha}$ for each $\alpha \geq-1$.

These examples suggest that a characterization of hyponormality of operators $S_{g} \in$ $\mathcal{L}\left(L_{a}^{2, \alpha}\right)$ may be difficult. On the other hand, a start has been made on the question of 
subdecomposability for the operators $S_{g} \in \mathcal{L}\left(H^{p}(\mathbb{D})\right)$ related to the Cesàro operator by compact perturbations. While local spectral properties are generally unstable under finite rank perturbations, [39, V.6.29], growth conditions do however behave reasonably under suitable perturbations.

Let $F$ denote the collection of finite rank operators. If $T \in \mathcal{L}(X)$, define $a_{n}(T)=$ $\inf \{\|T-A\|: \operatorname{dim} \operatorname{ran} A<n\}$ for each $n \geq 1$. Also, for every $r>0$, define $n_{T}(r)=$ $\inf \left\{n: a_{n+1}(T)<1 / r\right\}$. This is a measure of how well $T$ can be approximated by finite rank operators. With control on $n_{K}$, growth conditions as in Theorem 3.3 are inherited by $T+K$. The following is a version of $[29,2.3]$.

TheOREM 4.3. Suppose that $T \in \mathcal{L}(X), K \in \bar{F}$ and that there exist a closed disk $\nabla$ and a set $\mathcal{F} \subset \mathbb{C} \backslash \partial \nabla$ that accumulates only on a totally disconnected compact subset $E$ of $\partial \nabla$ such that

(1) $\sigma(T) \cup \sigma(T+K) \subseteq \mathcal{F} \cup \nabla$;

(2) $\sigma_{\ell}(T) \subseteq \mathcal{F} \cup \partial \nabla=: \mathcal{C}$, and

(3) $T$ has an analytic left resolvent $L$ on $\mathbb{C} \backslash \mathcal{C}$ such that $I-K L(\lambda)$ is invertible for all $\lambda \notin \mathcal{C}$.

Assume further that there exist a neighborhood $W$ of $\nabla$, a locally bounded function $\omega$ : $W \backslash E \rightarrow[0, \infty)$ and a decreasing function $f:(0, \infty) \rightarrow(0, \infty)$ such that

$$
\int_{0}^{1} \log ^{+} \log ^{+} f(t) d t<\infty \quad \text { and } \quad\|L(\lambda)\| \leq \omega(\lambda) f(\operatorname{dist}(\lambda, \mathcal{C}))
$$

for all $\lambda$ in $W \backslash \mathcal{C}$. If $K$ is such that

$$
\int_{0}^{1} \log ^{+} n_{K}(2 f(t)) d t<\infty,
$$

then $T+K$ has Bishop's property $(\beta)$.

As a consequence, we obtain the following, [29].

THEOREM 4.4. For each of the symbols $g(z)=1 /\left(1-z^{2}\right), g(z)=z^{m} /(1-z)$ and $g(z)=$ $f(z) /(1-z)$, for any function $f$ analytic in a neighborhood of $\overline{\mathbb{D}}$ with $\operatorname{Re}(f(z))>0$ for all $z \in \mathbb{D}$, the corresponding operator $S_{g}$ has Bishop's property $(\beta)$ on every $H^{p}, 1<p<\infty$.

The study of spectral and local spectral properties of generalized Cesàro operators is in its infancy. For functions $g$ other than the rational functions considered in this section, spectral properties of the corresponding generalized Cesàro operators are completely unknown, and nothing is known about subdecomposability beyond the specific examples of the last theorem. In particular, it is an open question whether $S_{g}$ has property $(\beta)$ on $H^{p}(\mathbb{D})$ for every rational function $g$.

Since $\operatorname{Re}\left(1 /\left(1-z^{2}\right)\right)>0$ and $\operatorname{Re}((1+z) /(1-z))>0$ for all $z \in \mathbb{D}$, the subdecomposable operators $S_{1 /\left(1-z^{2}\right)}$ and $S_{(1+z) /(1-z)}$ in $\mathcal{L}\left(H^{p}(\mathbb{D})\right), p>1$, are resolvents of generators of $\left(C_{0}\right)$ semigroups of weighted composition operators, [36]. Specifically, if $h_{t}(z)=$ $e^{-t} z / \sqrt{\left(e^{-2 t}-1\right) z+1}$, then the operators $U_{t} f(z)=h_{t}(z) / z f\left(h_{t}(z)\right)$ form a semigroup in $\mathcal{L}\left(H^{p}(\mathbb{D})\right)$ with generator $\mathcal{U} f(z)=\left(z^{2}-1\right)(z f(z))^{\prime}$, so $S_{1 /\left(1-z^{2}\right)}=R(0, \mathcal{U})$. In the case of the function $g(z)=(1+z) /(1-z)$, consider the Koebe function, $k(z)=z /(1-z)^{2}$, 
and define $k_{t}(z)=k^{-1}\left(e^{-t} k(z)\right)$. Then $V_{t} f(z)=k_{t}(z) / z f\left(k_{t}(z)\right)$ defines a semigroup in $\mathcal{L}\left(H^{p}(\mathbb{D})\right)$ with generator $\mathcal{V} f(z)=-1 / g(z)(z f(z))^{\prime}$. Just as for the semigroup associated with the Cesàro operator, it would be natural to investigate (local) spectral properties of these semigroups as well as those associated with other functions $g$ such that $\operatorname{Re}(g)>0$ on $\mathbb{D}$.

\section{References}

[1] E. Albrecht and J. Eschmeier, Analytic functional models and local spectral theory, Proc. London Math. Soc. (3) 75 (1997), 323-348.

[2] E. Albrecht, T. L. Miller and M. M. Neumann, Spectral properties of generalized Cesàro operators on Hardy and weighted Bergman spaces, preprint.

[3] A. Aleman and J. A. Cima, An integral operator on $H^{p}$ and Hardy's inequality, Journal d'Analyse Mathématique 85 (2001), 157-176.

[4] A. Aleman and A. G. Siskakis, An integral operator on $H^{p}$, Complex Variables Theory Appl. 28 (1995), 149-158.

[5] A. Aleman and A. G. Siskakis, Integration operators on Bergman spaces, Indiana Univ. Math. J. 46 (1997), 337-356.

[6] E. Berkson and H. Porta, Semigroups of analytic functions and composition operators, Michigan Math. J. 25 (1978), 101-115.

[7] E. Bishop, A duality theorem for an arbitrary operator, Pacific J. Math. 9 (1959), 379-397.

[8] A. Brown, P. Halmos and A. Shields, Cesàro operators, Acta Sci. Math. (Szeged) 26 (1965), 125-137.

[9] C. C. Cowen, Subnormality of the Cesàro operator and a semigroup of composition operators, Indiana Univ. Math. J. 33 (1984), 305-318.

[10] A. Dahlner, Decomposable extension of the Cesàro operator on the weighted Bergman space and Bishop's property $(\beta)$, in: Some Resolvent Estimates in Harmonic Analysis, Dissertation, Lund University, Lund, Sweden, 2003.

[11] J. A. Deddens, Analytic Toeplitz and composition operators, Canad. J. Math. 24 (1972), 859-865.

[12] M. Didas, $\mathcal{E}\left(\mathbb{T}^{n}\right)$-subscalar $n$-tuples and the Cesàro operator on $H^{p}$, Annales Universitatis Saraviensis, Series Mathematicae 10 (2000), 285-335.

[13] R. G. Douglas, On extending commutative semigroups of isometries, Bull. London Math. Soc. 1 (1969), 157-159.

[14] N. Dunford and J. Schwartz, Linear Operators, Part I, John Wiley and Sons, New York, 1958.

[15] I. Erdelyi and S. Wang, Equivalent conditions to the spectral decomposition property for closed operators, Trans. Amer. Math. Soc. 314 (1989), 171-186.

[16] J. Eschmeier and B. Prunaru, Invariant subspaces for operators with Bishop's property ( $\beta$ ) and thick spectrum, J. Funct. Anal. 94 (1990), 196-222.

[17] J. B. Garnett, Bounded Analytic Functions, Academic Press, New York, 1981.

[18] J. A. Goldstein, Semigroups of Operators and Applications, Oxford Mathematical Monographs, Clarendon Press, Oxford, 1985.

[19] G. H. Hardy, On a theorem of Hilbert, Math. Z. 6 (1920), 314-317.

[20] G. H. Hardy and J. E. Littlewood, Some properties of fractional integrals II, Math. Z. 34 (1932), 403-439. 
[21] H. Hedenmalm, B. Korenblum and K. Zhu, Theory of Bergman Spaces, Graduate Texts in Mathematics 199, Springer-Verlag, New York, 2000.

[22] E. Hille and R. S. Phillips, Functional Analysis and Semi-groups, Colloquium Publications 31, Amer. Math. Soc., Providence, Rhode Island, 1957.

[23] T. Ito, On the commutative family of subnormal operators, J. Fac. Sci. Hokkaido Univ. (Sapporo) 14 (1958), 1-15.

[24] T. L. Kriete and D. Trutt, The Cesàro operator on $\ell^{2}$ is subnormal, Amer. J. Math. 93 (1971), 215-225.

[25] K. B. Laursen and M. M. Neumann, An Introduction to Local Spectral Theory, Clarendon Press, Oxford, 2000.

[26] J. Miao, The Cesàro operator is bounded on $H^{p}$ for $0<p<1$, Proc. Amer. Math. Soc. 116 (1992) 4, 1077-1079.

[27] T. L. Miller and V. G. Miller, On the approximate point spectrum of the Bergman space Cesàro operator, Houston J. Math. 27 (2001), 479-494.

[28] T. L. Miller and V. G. Miller, The Cesàro operator on the Bergman space $A^{2}(\mathbb{D})$, Arch. Math. (Basel) 78 (2002), 409-416.

[29] T. L. Miller, V. G. Miller and M. M. Neumann, Growth conditions, compact perturbations and operator subdecomposability, with applications to generalized Cesàro operators, J. Math. Analysis Appl., to appear.

[30] T. L. Miller, V. G. Miller and R. C. Smith, Bishop's property $(\beta)$ and the Cesàro operator, J. London Math. Soc. (2) 58 (1998), 197-207.

[31] Ch. Pommerenke, Schlichte Funktionen und analytische Funktionen von beschränkter mittlerer Oszillation, Comment. Math. Helv. 52 (1977), 591-602.

[32] G. Prajitura, The density of the hypercyclic operators in the strong operator topology, Integral Equations Operator Theory 49 (2004), 559-560.

[33] M. Putinar, Hyponormal operators are subscalar, J. Operator Theory 12 (1984), 385-395.

[34] W. Rudin, Functional Analysis, 2nd ed., McGraw-Hill, New York, 1991.

[35] A. G. Siskakis, Composition semigroups and the Cesàro operator on $H^{p}$, J. London Math. Soc. (2) 36 (1987), 153-164.

[36] A. G. Siskakis, The Koebe semigroup and a class of averaging operators on $H^{p}(\mathbb{D})$, Trans. Amer. Math. Soc. 339 (1993), 337-350.

[37] A. Siskakis, Semigroups of composition operators in Bergman spaces, Bull. Austral. Math. Soc. 35 (1987), 397-406.

[38] A. G. Siskakis, On the Bergman space norm of the Cesàro operator, Arch. Math. (Basel) 67 (1996), 312-318.

[39] F.-H. Vasilescu, Analytic Functional Calculus and Spectral Decompositions, Editura Academiei and D. Reidel Publishing Company, Bucharest and Dordrecht, 1982.

[40] S. Wang, Abel ergodic properties and spectral decomposition for locally integrable semigroups, J. Math. Anal. Appl. 231 (1998), 116-131.

[41] S. W. Young, Algebraic and Spectral Properties of Generalized Cesàro Operators, Dissertation, University of North Carolina, Chapel Hill, 2002.

[42] S. W. Young, Generalized Cesàro operators and the Bergman space, J. Operator Theory, to appear. 
